\title{
Interplay between structure and superconductivity: Metastable phases of phosphorus under pressure
}

\author{
José A. Flores-Livas, ${ }^{1}$ Antonio Sanna, ${ }^{2}$ Alexander P. Drozdov, ${ }^{3}$ Lilia Boeri, ${ }^{4,5}$ Gianni Profeta, ${ }^{6}$ \\ Mikhail Eremets, ${ }^{3}$ and Stefan Goedecker ${ }^{1}$ \\ ${ }^{1}$ Department of Physics, Universität Basel, Klingelbergstrasse 82, 4056 Basel, Switzerland \\ ${ }^{2}$ Max-Planck Institut für Microstructure Physics, Weinberg 2, 06120 Halle, Germany \\ ${ }^{3}$ Max-Planck Institut für Chemie, Chemistry and Physics at High Pressures Group Postfach 3060, 55020 Mainz, Germany \\ ${ }^{4}$ Institute of Theoretical and Computational Physics, Graz University of Technology, NAWI Graz, 8010 Graz, Austria \\ ${ }^{5}$ Dipartimento di Fisica, Sapienza Università di Roma, 00185 Rome, Italy \\ ${ }^{6}$ Dipartimento di Fisica Università degli Studi di L'Aquila and SPIN-CNR, I-67100 L'Aquila, Italy
}

(Received 19 April 2017; published 20 July 2017)

\begin{abstract}
Pressure-induced superconductivity and structural phase transitions in phosphorus $(\mathrm{P})$ are studied by resistivity measurements under pressures up to $170 \mathrm{GPa}$ and by fully $a b$ initio crystal structure exploration and superconductivity calculations up to $350 \mathrm{GPa}$. Two distinct superconducting transition temperature $\left(T_{C}\right)$ vs pressure $(P)$ trends at low pressure have been reported more than 30 years ago, and we are able to devise a consistent explanation founded on thermodynamically metastable phases of black phosphorus. Our experimental and theoretical results form a single, consistent picture which not only provides a clear understanding of elemental $\mathrm{P}$ under pressure but also sheds light on the longstanding and unsolved anomalous superconductivity trends. Moreover, at higher pressures we predict a similar scenario of multiple metastable structures which coexist beyond their thermodynamical stability range. We observe that all the metastable structures systematically exhibit larger transition temperatures than the ground-state structures, indicating that the exploration of metastable phases represents a promising route to design materials with improved superconducting properties.
\end{abstract}

DOI: 10.1103/PhysRevMaterials.1.024802

\section{INTRODUCTION}

The discovery that sulfur hydride $\left(\mathrm{SH}_{3}\right)$ is a superconductor with a record-breaking critical transition temperature $\left(T_{C}\right)$ of $200 \mathrm{~K}$ [1] has disproved a decades-long prejudice against high $T_{C}$ occurring conventionally [2,3]. This result demonstrated that extreme pressures represent an avenue to access new physical phenomena and exotic states of matter, which in the next years may lead to many surprises. Sulfur hydride is not an isolated example of conventional high- $T_{C}$ superconductivity at high pressures [4-13], since a few months later also phosphine $\left(\mathrm{PH}_{3}\right)$ was observed to superconduct at transition temperatures as high as $100 \mathrm{~K}$ at $200 \mathrm{GPa}$ [14]. In this case, experimental and theoretical evidences indicate that superconductivity most likely involves a metastable structure of phosphorus hydride, stabilized by a particular, reproducible experimental condition $[15,16]$.

Metastable phases, which can be accessed only under specific thermodynamical conditions, play a major role in determining the high-pressure properties in many compounds; in some cases, they can also lead to complicated superconducting phase diagrams, even for simple elements [17]. The most prominent example is hydrogen, where the search for a possible metallic phase [18] which could be a room-temperature superconductor [19] has been going on for decades. Actual hints for metallization have been recently reported in Ref. [20] and were based on the temperature dependence of electrical resistance and disappearance of Raman spectra. However, the reported metallization in Ref. [21] observed for atomic hydrogen based on reflectance of the sample is still highly debated [22-24]. What is certain is that the metallic phase involves metastable phases that are only accessible at high pressures and precise temperatures [20,25-27].
Even though its critical temperature is less spectacular than those of hydrogen and hydrides, phosphorus exhibits one of the richest phase diagrams of all elements, both at ambient [28,29] and high pressures. In particular, in this work, combining high-pressure experiments with a complete $a b$ initio characterization, we show that structural metastability plays a crucial role in determining the superconducting phase diagram, which is unquestionably driven by pressure and temperature conditions. Our $a b$ initio study allowed us to identify the structural and superconducting properties of several metastable high-pressure phases that we show have systematically larger transition temperatures than the putative ground-state ones.

\section{RESULTS}

The superconducting phase diagram of $\mathrm{P}$ is intriguingly complicated; the top panel of Fig. 1 compares the previous experimental results (gray symbols and lines) with our experiments (black symbols and lines). The first accurate resistivity measurements on P under compression date back to 1985 and were performed by Kawamura et al. [30]. The authors reported an anomalous behavior in the superconducting transition temperatures up to pressures of $30 \mathrm{GPa}$ : the value of $T_{C}$ was found to strongly depend on the $P-T$ path followed in experiment. The problem was revisited by Karuzawa et al. [31] in early 2000s, with a second set of experiments in which transition temperatures were measured for pressures up to $97 \mathrm{GPa}$, producing a single $T_{C}$ trend. More recently Guo et al. [32] carried out Hall electrical measurements under pressure up to $50 \mathrm{GPa}$ and reported a nonmonotonic trend of $T_{C}$ at low pressures and an anomaly due to a Lifshitz transition at $\sim 17 \mathrm{GPa}$. 


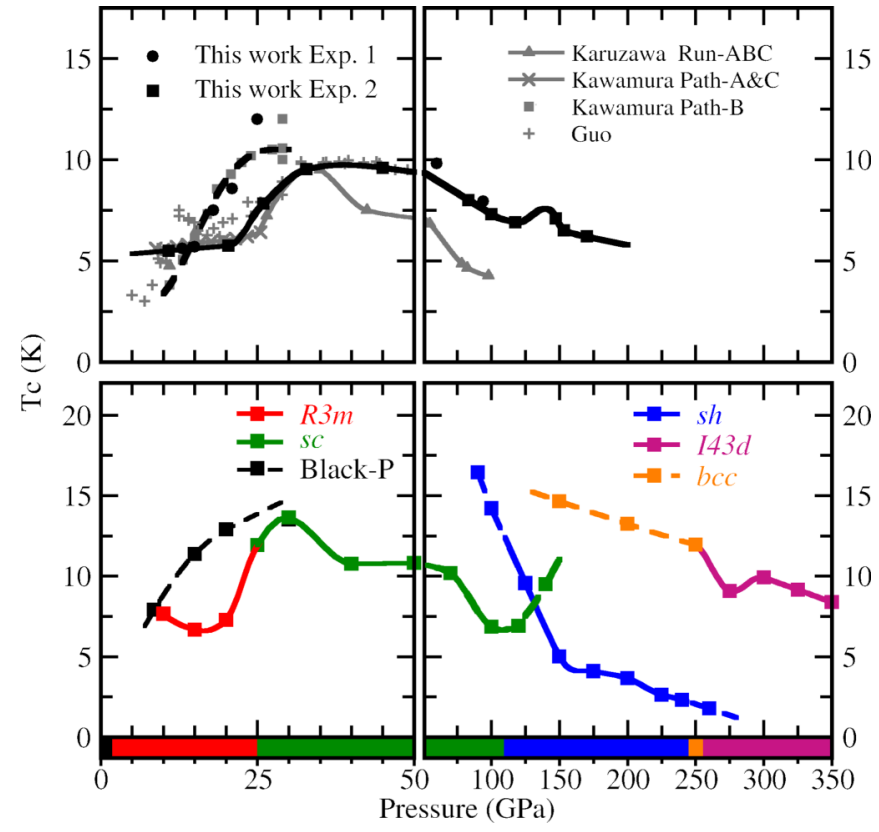

FIG. 1. Summary of all the critical temperatures observed experimentally [30-32] (top panels) and calculated theoretically in this work (bottom panels). In the experimental panels the dashed and full lines are guidelines to the eye and are used to distinguish the trend that we interpret as due to metastable black-P (dashed) from the energetically stable sequence of transformations (see text). The color bar at the bottom indicates the sequence of calculated ground-state structures; the color code is explained in the legend of the bottom panels.

Clearly, despite the effort and several experiments over decades, the explanation of the superconducting trends and more importantly the anomalous dependence on thermodynamic conditions remains unsolved. Given the polymorphism of elemental phosphorus already at ambient conditions, one could speculate that the anomalous $T_{C}$ vs $P$ trends are caused by the coexistence of metastable phases. Therefore, in the present work we design two different sets of experiments which specifically aim at accessing different phases (stable and metastable) following two $P-T$ thermodynamic paths, as schematically illustrated in the top panels of Fig. 2.

In the first set, Exp. 1 (the measured $T_{C}$ is reported as black circles in the top panel of Fig. 1), the sample is constantly kept at low temperature while pressure is increased. In the second $P-T$ path (Exp. 2) the temperature is raised at higher temperatures when applying pressure and for resistivity measurements. In this figure the bottom panels show the resistivity measurements at different pressures and $T_{C}$ values are indicated with arrows. Clearly the $T_{C}$ vs $P$ behavior of the two datasets is rather different. In the first set (black circles in Fig. 1) a slow increase of $T_{C}$ up to $25 \mathrm{GPa}$ is observed, while for the second set of experiments $T_{C}$ sharply increases with pressure (black squares in Fig. 1). The two data sets merge at $25 \mathrm{GPa}$, and a single trend is observed up to the highest common pressure measured ( $94 \mathrm{GPa}$ ).

\section{A. Ab initio phase diagram of $P$ under pressure}

Figure 3 shows the computed enthalpy for different allotropes of phosphorus under pressure found using our crystal
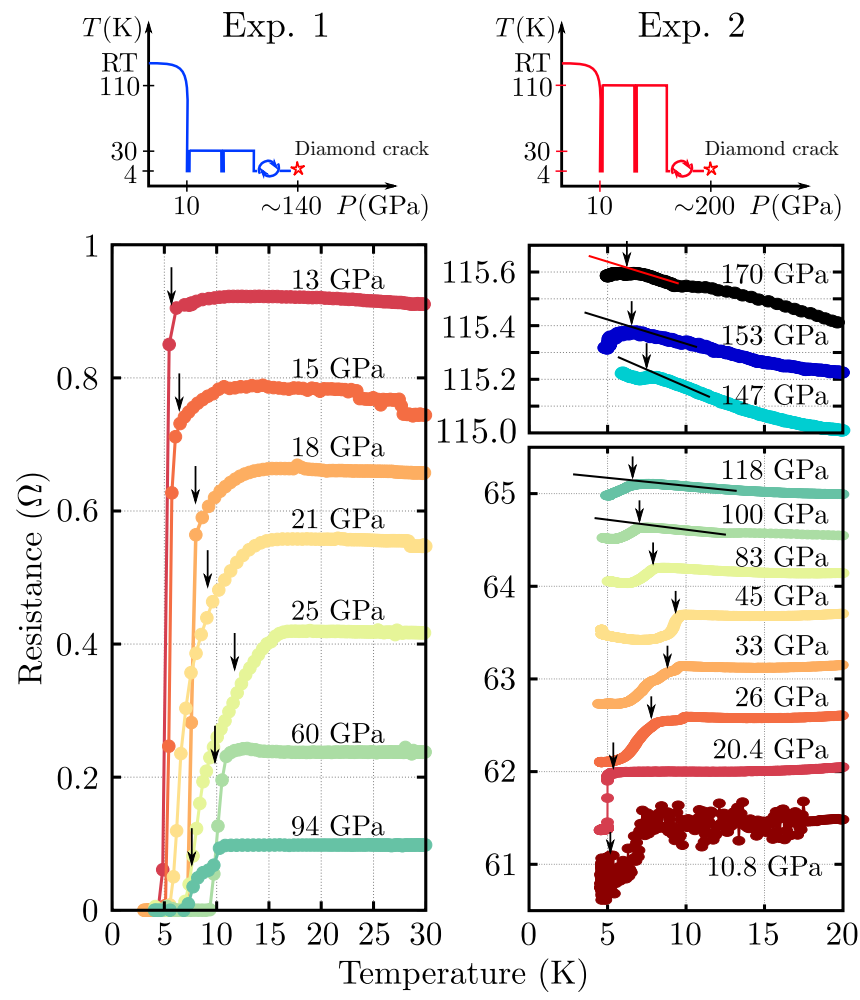

FIG. 2. Bottom panels show resistivity measurements as a function of temperature for different pressure conditions: In Exp. 1 (top-left panel) the sample was cycled at low temperature $(<30 \mathrm{~K})$ and in Exp. 2 (top-right panel) it was cycled up to high temperature $(<260 \mathrm{~K})$. The $T_{C}$ onsets for each pressure are marked with arrows. The corresponding values are summarized in Fig. 1.

structure prediction method. The enthalpy difference is shown with respect to the $s c(P m-3 m)$ phase (crystal structures are shown in Fig. 4). The lowest-enthalpy sequence of transitions, according to the calculations, is the following: Red-P (triclinic $P-1)[33]$ is stable at $0 \mathrm{GPa}$ and almost degenerate with black-P (Cmca) [34], which is the experimentally observed phase; phase $P-I I(A 7-R 3 m)$ occurs from 3 to $16 \mathrm{GPa}$. The simple cubic $(s c-P-I I I)$ lattice dominates for pressures up to $120 \mathrm{GPa}$, where the simple hexagonal $(s h)$ lattice $(s h-P 6 \mathrm{mmm})$ is stable up to $\sim 225 \mathrm{GPa}$. We find the $b c c(I m-3 m)$ crystal stable from 225 to $250 \mathrm{GPa}$, and finally the last phase $(I-43 d)$ is stable from 250 to beyond $350 \mathrm{GPa}$. The sequence of transitions is shown as a color bar at the bottom of Fig. 1.

The three relevant pressure intervals in which phase transitions take place are highlighted in the top panels of Fig. 3. The first one (left) shows the low-pressure regime. At ambient conditions phosphorus is known in at least three different allotropic forms: black-P, red-P, and white-P. Experimentally, black-P is the most stable form [34,35], which transforms to the $A 7$ phase [36] for pressure above $5 \mathrm{GPa}$ [37]. In our calculations, red-P is the ground state at zero pressure (although is almost degenerate with black-P), while the stabilization energy of white- $\mathrm{P}$ is $130 \mathrm{meV}$ per atom higher (or equivalently, $\sim 1500 \mathrm{~K}$ ). The apparent disagreement is due to the fact that the standard generalized gradient approximation 

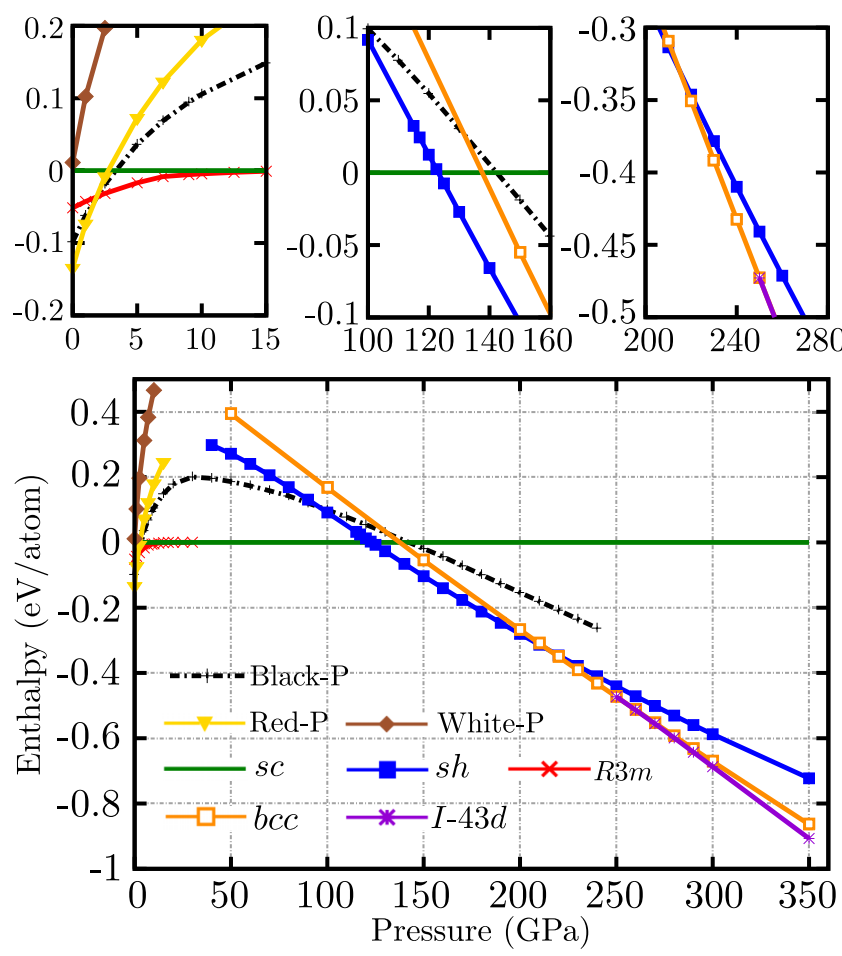

FIG. 3. Calculated enthalpy for different crystal structures of phosphorus with respect to $s c$ as a function of pressure. The top panels show an enlargement of three relevant pressure windows in which several structures are energetically competitive within orders of $\sim 100 \mathrm{meV}$ (left), $\sim 15 \mathrm{meV}$ (center), and $\sim 5 \mathrm{meV}$ (right).

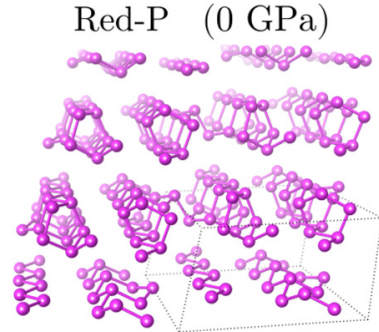

Black-P

(15 GPa)

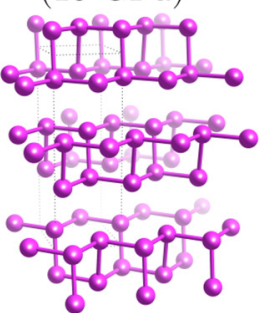

sh

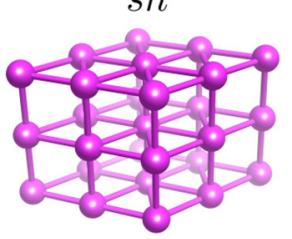

(120 GPa)

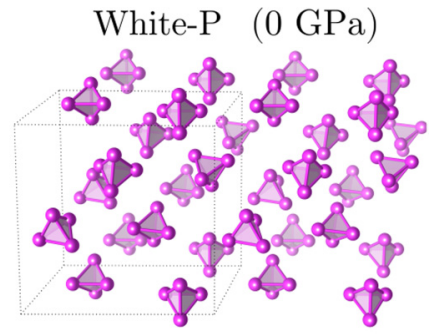

$s c$ $(50 \mathrm{GPa})$
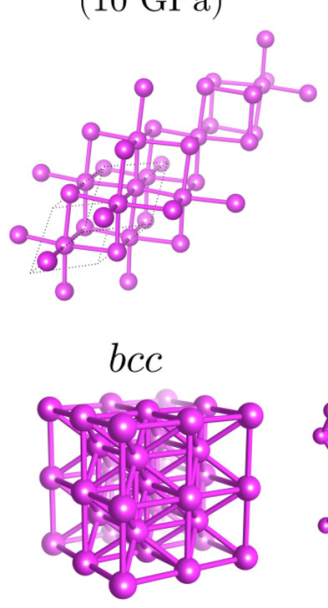

(250 GPa)

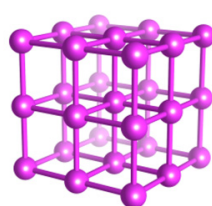

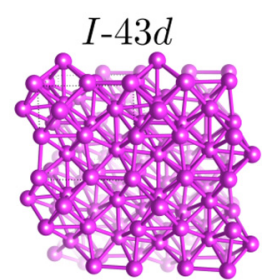

(300 GPa)
FIG. 4. Crystal structures of phosphorus at indicated pressures.
(GGA) functional used in this work is inaccurate for layered (van der Waals bonded) or polymeric systems in predicting the exact structural sequence for different polymorphisms. However, although the van der Waals interaction is rather important in the very low-pressure region of the phase diagram (0-5 GPa), it plays only a minor role at higher pressures, where superconductivity occurs. We find that black-P remains enthalpically competitive within a comparable order of magnitude $(\sim 0.1 \mathrm{eV})$ with other low-enthalpy phases up to pressures as large as $30 \mathrm{GPa}$. As discussed in detail in the next section, the metastability of this phase turns out to be fundamental to describe the experimental trend in $T_{C}$.

\section{B. High-pressure experiments}

The second panel (top central) of Fig. 3 shows the pressure interval in which the $s h \rightarrow b c c$ transition occurs. In this window $s c$, sh (P6mmm), IM-Cmmm (not shown), bcc, and surprisingly, black-P (in a collapsed form) are structures that are all accessible within a few tens of meV energy difference. This enthalpy landscape is consistent with experimental evidences that in this pressure range the $s h \rightarrow b c c$ transition occurs via an intermediate incommensurate phase [38,39].

A third pressure range worth analyzing in more detail (top right panel) is where the $s h$ and $b c c$ enthalpy curves cross each other and the $b c c$ to $I-43 d$ transformation occurs $(I-43 d$ is a distorted form of $b c c)$. Note that in this case the enthalpy differences between all three phases are extremely small, i.e., within the computational accuracy, so that vibrational entropy corrections (not included) and stabilization of distorted complex structures [36,40] could in principle affect the energetic ranking of the structures and the transition pressures. However, these corrections will shift the transition pressure by no more than $\sim 5 \mathrm{GPa}$, which is below the experimental error to estimate the pressure $(\sim 10 \mathrm{GPa})$.

Indeed, our $a b$ initio zero-temperature phase diagram is in substantial agreement with the sequence experimentally observed [36-39,41-46] and is therefore a good starting point to calculate the superconducting critical temperatures as a function of the pressure. Furthermore, our analysis has allowed us to characterize several of the structural transitions as first order, i.e., with discontinuous $P(V)$ behaviors (see Fig. 5); this could lead to a possible path to stabilize metastable structures under suitable thermodynamic conditions.

\section{Ab initio predicted superconducting temperatures}

Using state-of-the-art density functional theory for superconductors (SCDFT) combined with density-functional perturbation theory for the calculation of phonon dispersions and electron-phonon coupling and linear response theory in the random phase approximation for the evaluation of electronelectron repulsion, we have computed the superconducting properties (anomalous density and critical temperature, $T_{C}$ ) for all the identified structures of $\mathrm{P}$ which are dynamically stable. The corresponding $T_{C}$ values are shown in the lower panel of Fig. 1 as full (dashed) lines for ground-state (metastable) structures.

Low pressures $(0-50 \mathrm{GPa})$. As shown in the previous section, phosphorus has an extremely rich phase diagram of 


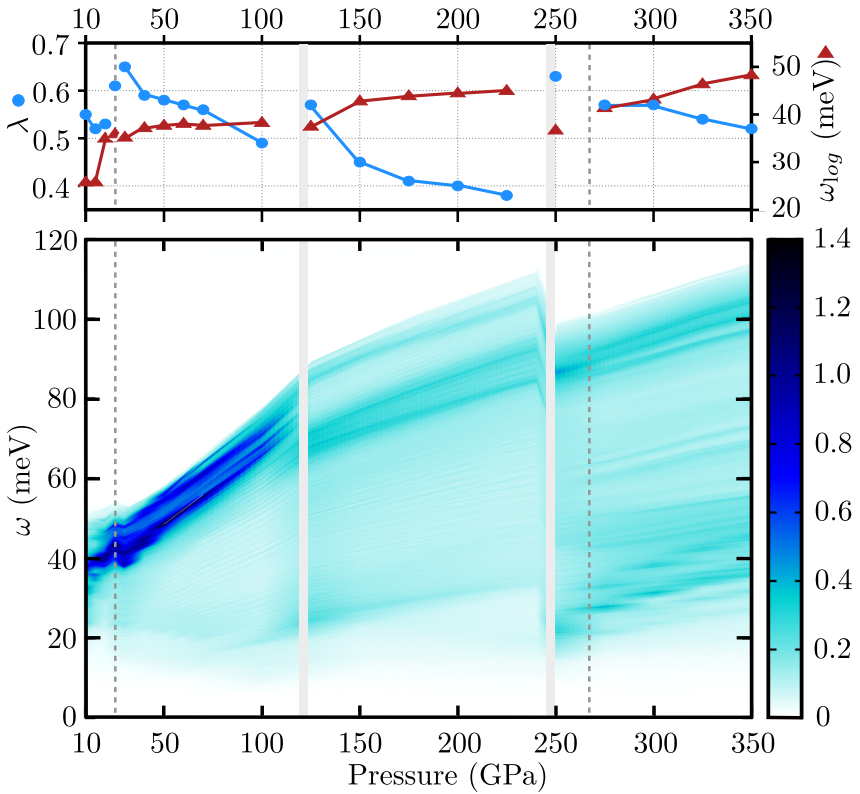

FIG. 5. Bottom panel shows the pressure evolution of the Eliashberg function $\alpha F^{2}(\omega)$ [Eq. (1)] for all the thermodynamically stable phases of phosphorus between 10 and $350 \mathrm{GPa}$. Pressures are shown in abscissas, frequencies $(\omega)$ in ordinates; the function value is given by the color scale. White vertical gaps mark first-order phase transitions and gray dashed lines mark second-order transitions. Top panel shows the electron-phonon coupling constant $\lambda$ (left $y$ axis) and the characteristic phonon energy $\omega_{\log }$ (right $y$ axis) obtained from the Eliashberg spectral functions (see Methods and Supplemental Materials [50]).

unique complexity; at ambient conditions of pressure and temperature its three most common polymorphs, black-P, red-P, and white-P, are semiconducting or insulating. While black-P and red-P achieve metallization within a few GPa [estimating the exact value would require calculating corrections to the band gap beyond density functional theory (DFT)], white-P is still insulating at $7 \mathrm{GPa}$ with a band gap of about $1.4 \mathrm{eV}$. Considering that DFT typically underestimates band gaps in insulators, this implies that white-P cannot be considered a candidate for the superconducting phase and therefore will not be included in further investigations. Also red-P is excluded as a possible superconducting phase in the low-pressure range, because, although dynamically stable for 10 and $15 \mathrm{GPa}$, the calculated $T_{C}$ is too low $(<1 \mathrm{~K})$ to account for any experimental evidence of superconductivity. Upon further compression our calculations shown that red-P collapses to a simple cubic lattice. For black-P, on the contrary, the calculated $T_{C}$, depicted as black line in Fig. 1, rapidly increases as a function of pressure. This a consequence of the relatively strong electron-phonon coupling, which is consistent with previous predictions on doped black-P and phosphorene [47]. This superconducting structure of black-P, which remains energetically metastable and dynamically stable for pressures up to $20 \mathrm{GPa}$, with transition temperatures as high as $\simeq 13 \mathrm{~K}$, is actually a modulated superstructure of the true black-P, obtained by supercell relaxation along soft directions. Upon further compression the modulated black-P relaxes to the simple cubic lattice.
However, at pressures in which superconductivity $\operatorname{occurs}(\sim 10 \mathrm{GPa})$ the ground-state structure is not black-P but $A 7-\mathrm{P}$, in good agreement with $\mathrm{x}$-ray diffraction (XRD) measurements [40] and previous theoretical calculations [48]. In this phase the calculated evolution of $T_{C}$ is pressure independent; $T_{C}$ does remains constant $\sim 7 \mathrm{~K}$ (red line in bottom panel in Fig. 1). Noticeably, we find that the sudden increase in $T_{C}$ for pressures above $20 \mathrm{GPa}$ occurs in correspondence to the $A 7 \rightarrow s c$ second-order phase transition. Our calculations show that this substantial increase in $T_{C}$ is not originated, as one could expect, from soft phonons inducing the structural transition, which could consequently provide large electron-phonon coupling, but is instead triggered by an electronic Lifshitz transition [49]. It does happen indeed that, upon increasing pressure, an additional band crosses the Fermi level, contributing to the electronic density of states (DOS) and providing a strong interband scattering channel that reinforces the Cooper pairing. An extensive analysis of this effect is provided in the Supplemental Material [50] and link. The same behavior was recently predicted also in the superconducting properties of elemental sulfur under pressure [51], which remarkably has many common aspects with phosphorus.

Once the Lifshitz transition is completed, $T_{C}$ marginally decreases with pressure as the occupation of the additional band increases, enhancing the electronic screening of the electron-phonon coupling. As a consequence, $T_{C}$ results are rather featureless in the range between 40 and $100 \mathrm{GPa}$, where the only relevant phase is the $s c$ one, showing $T_{C}$ values decreasing with pressure, in agreement with previous estimation [48]. Considering only the thermodynamically stable structures, the predicted $T_{C}$ closely follows the pressure dependence of the BCS-type coupling $\lambda[\mathrm{Eq}$. (2)] reported in Fig. 5 (blue curve in top panel). This is due to the combination of three factors: First, superconductivity is happening in the "weak-coupling limit," since the values of $\lambda$ are between 0.4 and 0.7. In this limit $T_{C}$ is extremely sensitive to $\lambda$. Second, the characteristic phonon energy scale $\omega_{\log }$ [Eq. (3) and red line in the upper panel of Fig. 5] shows almost no pressure dependence, as would occur in the presence of phononic instabilities. Third, the repulsive Coulomb interaction between electrons in the Cooper pairs, represented by the Coulomb pseudopotential parameter $\mu^{*}[38,52]$, is constant and $\simeq 0.1$ for all structures and pressures, which is rather typical for $s p$-bonded systems. More insight on the pressure evolution of the characteristic $e p$ coupling parameters for the thermodynamically stable phases can be obtained by analyzing the pressure evolution of the electron-phonon spectral function $\alpha^{2} F(\omega)$, shown in the bottom panel of Fig. 5. In the plot, frequencies are shown in ordinates, pressures in abscissas, and the color scale indicates the intensity of the $\alpha^{2} F(\omega)$; strongly coupled phonons appear as darker regions at the corresponding frequencies. In general, the maximum phonon frequencies raise as pressure is increased. Up to around 20 $\mathrm{GPa}$, the coupling is concentrated in the high-frequency modes while the distribution changes at higher pressures $(25-50 \mathrm{GPa})$, where it appears more evenly distributed. This means that, despite the pronounced hardening of the maximum phonon frequencies with pressure, the center of mass of the spectrum remains almost constant, and there are no signatures of lattice instabilities induced by electron-phonon coupling. 
In contrast to the weak-coupling behavior of thermodynamically stable phases, metastable phases (black-P, $s c$, sh, and $b c c$ ) exhibit a marked strong-coupling behavior at the limit of their dynamical stability range. For these systems, upon approaching their structural transition, a considerable part of the phonon spectral weight is shifted to low frequencies, consequently increasing $\lambda$ and a simultaneously decreasing $\omega_{\log }$. Further details, including the calculated $\alpha^{2} F(\omega), \lambda, \omega_{\log }$ and the Coulomb screening parameters for all phases discussed in this work can be found in the Supplemental Material [50].

High pressures (50-350 GPa). We will now analyze the high-pressure range, for which our calculated $T_{C}$ values are summarized in the right bottom panel of Fig. 1. In the $s c$ phase, $T_{C}$ continues to decrease until the $s c$-to-sh transition occurs. Since this is a transition of first order, as the one from black-P to $s c$, we investigated the metastability of the two phases across their thermodynamic boundaries. For higher and lower pressures and for both systems our calculations predict dynamically stable structures: $s c$ up to $150 \mathrm{GPa}$ and $s h$ down to $80 \mathrm{GPa}$. Away from their thermodynamical phase boundaries, $T_{C}$ varies rapidly in both systems, and this is due to a phonon softening that lowers the phonon energy and increases the coupling. From 150 to $250 \mathrm{GPa}$, the $T_{C}$ of $s h$-P decreases steadily below $3 \mathrm{~K}$, and this phase is therefore moderately interesting for superconductivity. On the other hand, the $b c c / I-43 d$ sequence of structures, stable above 250 $\mathrm{GPa}$, is more promising. In fact, these phases show a larger $T_{C}$, well above $10 \mathrm{~K}$. It is also worth mentioning that between 150 and $250 \mathrm{GPa}$ the $b c c$ structure, which is thermodynamically metastable, exhibits transition temperatures 3 times larger than those calculated for the $s h$ structure.

\section{DISCUSSION}

One of the crucial points of this work is to identify metastability as a distinctive feature of the phase diagram of phosphorus. In this element, the presence of several discontinuous phase transitions, not directly triggered by phononic instabilities, implies the coexistence of several metastable phases with distinct superconducting properties across the phase diagram. This means that the initial conditions and the $P-T$ path followed in the experiments largely determine the resulting crystal structure, coexistence of phases, and the possibility of observing high- $T_{C}$ metastable phases.

Using high-pressure experiments and first-principles calculations we are able to draw a consistent picture of the full phase diagram up to $350 \mathrm{GPa}$. We propose that the coexistence of two distinct superconducting phases of phosphorus in the 10-30 GPa pressure window successfully accounts for the the "anomalous" $T_{C}$ vs $P$ trend first observed by Kawamura and co-workers [30]. Furthermore, we identified theoretically two other pressure regions in which superconducting $T_{C}$ anomalies could be observed depending on the experimental conditions.

We first discuss the 10-30 GPa pressure interval, for which several experimental data are available. Here, we hypothesize that three phases actually play a role in the superconducting phase diagram, namely, black-P, $R 3 m$, and $s c$, and that the bifurcation in $T_{C}$ seen by experiments is due to the coexistence the ground-state low- $T_{C} A 7-\mathrm{P}$ and high- $T_{C}$ metastable black$\mathrm{P}$ phases, which both collapse to $s c$ at $P \approx 20 \mathrm{GPa}$. Our hypothesis perfectly accounts for the existing literature results of Kawamura et al. [30], in which the pressure cell was initially loaded with black-P and pressure was subsequently increased, either keeping the system at low temperature (Kawamura, path $\mathrm{B}$ in Fig. 1) or following a room-temperature path (paths A and C). The first experimental procedure leads to the highest $T_{C}$ and is remarkably well reproduced by calculations for black-P, while the second leads to a low $T_{C}$ and matches the calculated trend for $A 7$ phosphorus.

The two sets of experiments in this work were explicitly designed to reproduce the distinct $T_{C}$ trends and to test our interpretation in terms of $A 7$ and black phosphorus. In contrast to previous experiments, in this work the cell was loaded with red-P at room temperature. In the first set (Exp. 1), the samples were kept at low temperatures during the whole pressure run. The first pressure measured was high enough ( $\sim 5 \mathrm{GPa})$ to ensure a complete transition of red-P to black-P; according to our calculations, the transition should occur at $\sim 2.5 \mathrm{GPa}$, and black-P should survive as a metastable phase for pressures well above $20 \mathrm{GPa}$, where it collapses to the $s c$. On the contrary, following the second path in the $P-T$ phase space (Exp. 2), where annealing cycles allow the thermodynamically ground-state structures to be reached, will stabilize the $A 7$ phase between 5 and $\sim 20 \mathrm{GPa}$. Upon further compression the $A 7$ structure will transform continuously to $s c$, as our crystal prediction method confirmed. The measured $T_{C}$ vs $P$ trends for the two paths closely reproduce the corresponding measurements of Kawamura et al. and are in excellent agreement with our theoretical predictions, supporting our interpretation that the high- $T_{C}$ phase is metastable black-P. An alternative explanation would be to ascribe the high- $T_{C}$ curve in Exp. 1 to a metastable red-P phase. We can safely rule out this hypothesis because, according to our calculations, after the metallization red-P has a negligible $T_{C}(<1 \mathrm{~K})$.

It is worthwhile to comment on the recent measurements by Guo et al. [32]. They extend up to $50 \mathrm{GPa}$, and are generally consistent with previous measurements and ours, but they seem to contradict our understanding of the low-pressure range. In fact, the authors report a gradual increase in $T_{C}$ from 5 to $10 \mathrm{GPa}$ followed by an abrupt jump, which does not have a straightforward interpretation. Our theoretical results suggest that a possible explanation for this phenomenon is the existence of a mixed black-P-R3m $(\mathrm{O}+\mathrm{R})$ phase (suggested by the same authors) which prevents a clear observation of the two distinct $T_{C}$ trends and is probably responsible for the oscillations in $T_{C}$ observed up to $\sim 25 \mathrm{GPa}$, where the samples enter to the $s c$ phase and Guo's data merge with our measurements and older data.

Besides the low-pressure region already explored by Kawamura et al., we predict two other ranges of pressure in which different crystal structures are energetically accessible and experimentally measurable: the first is around $\sim 110$ $\mathrm{GPa}$, and a second is above $\sim 220 \mathrm{GPa}$. In the first case, unfortunately the two competing phases have very similar critical temperatures (see $T_{C}$ for hex and $s c \approx 120 \mathrm{GPa}$ ) and are thus hard to distinguish experimentally. Moreover, phonons are softening close to the transition, coming both from low and high pressures, indicating that it could be difficult to stabilize metastable structures and that complex disordered or modulated structures may form [38]. In the 
second case, the $T_{C}$ 's of the ground-state and metastable structures (which according to our predictions is of comparable energetics at the relevant pressures) differ by a factor of 3 (see Fig. 3 and $T_{C}$ 's for $s h, b c c$, and $I-43 d$ above $\sim 225 \mathrm{GPa}$ ). Therefore, these two distinct $T_{C}$ vs $P$ trends should be easily discernible by experiments. However, no superconductivity measurements have been reported yet at these pressures, which was indeed unaccessible also for our current experimental setup. Nevertheless, we note that, remarkably, our theoretical prediction of phase coexistence is consistent with recent XRD experiments [40]. A possible path to achieve the synthesis of these metastable phases would be to start from the ground-state structure at high pressures $I-43 d(\lesssim 270 \mathrm{GPa})$, cycle the sample trough temperatures high enough to overcome the energetic barrier to the $s h$ phase, and then cool the sample slowly releasing the pressure down to $230 \mathrm{GPa}$.

In summary, we have conducted a systematic theoretical and experimental investigation of elemental phosphorus under pressure. The excellent agreement between our experimental and theoretical results allowed us to not only reconcile previous unexplained anomalies, but also to shed light onto the complex behavior of metastable phases under pressure. Elemental phosphorus, like other elements [53] and compounds [54], has the tendency to form many polymorphs that differ substantially in their electronic and superconducting properties and can coexist in metastable forms over different pressures. In this work, we showed that many of these exhibit critical temperatures which are distinctively higher than the putative ground-state structures. This suggests that the selective stabilization of metastable phases represents, in the near future, a viable strategy to improve the superconducting properties of conventional superconductors.

\section{METHODS}

Experimental procedure. High-pressure electrical measurements were carried out using a diamond anvil cell (DAC) with an anvil tip diameter of 200-300 mm bevelled at 7-8 deg and with culet surface between 40 and $80 \mathrm{~mm}$. Four Ti electrodes were sputtered on the diamond anvil for the first experiment (Exp. 1) and three $\mathrm{Ti}$ electrodes for the second experiment (Exp. 2). The electrodes were capped with $\mathrm{Au}$ to prevent oxidation of the Ti. (A zero contribution by the diamond surface to the conductivity was checked.) An insulating gasket of Teflon was used to separate the metallic gasket from the electrodes. Red phosphorus was loaded in the DAC at ambient temperature and clamped at these conditions. The first pressure point after being clamped was already about 3-5 GPa in both experiments. Then the DAC was placed into a cryostat and cooled down to measure the first point of $T_{C}$ at $13 \mathrm{GPa}$ for the first experiment (Exp. 1) and $11 \mathrm{GPa}$ for the second experiment (Exp. 2). The pressure was determined by a diamond edge scale at low temperatures using Raman spectra and we monitored any possible Raman signal from the samples. We used the 632.8-nm line of a He-Ne laser to excite the Raman spectra measured with a Raman spectrometer equipped with a nitrogen-cooled CCD and notch filters with resolution better than $2 \mathrm{~cm}^{-1}$. Two different conditions were tested in our experiments, Exp. 1 and Exp. 2 as summarized in Fig. 2, with the only difference being the increased temperature at the beginning of the experiment. All the resistance measurements were done in increasing pressure; we could not perform measurements under decompression due to the diamond culet crack.

\section{A. Crystal phase diagram exploration}

To sample the enthalpy landscape we employed the minima hopping method (MHM) [55] with unit cells of up to eight atoms for selected pressures in the range of 0-350 $\mathrm{GPa}$. This method has been successfully used for global geometry optimization in a large variety of applications [56-60], including superconducting materials at high pressure [58]. The MHM was designed to thoroughly scan the low-lying enthalpy landscape of any compound and identify stable phases by performing consecutive short-molecular-dynamics escape steps followed by local geometry relaxations. The enthalpy surface is mapped out efficiently by aligning the initial molecular dynamics velocities approximately along soft-mode directions [61,62], thus exploiting the Bell-EvansPolanyi [63] principle to steer the search towards low-energy structures. Energy, atomic forces, and stresses were evaluated at the DFT level with the Perdew-Burke-Ernzerhof (PBE) [64] parametrization to the exchange-correlation functional. A plane-wave basis set with a high cutoff energy of $1000 \mathrm{eV}$ was used to expand the wave function together with the projector augmented wave (PAW) method as implemented in the Vienna Ab Initio Simulation Package VASP [65]. Geometry relaxations were performed with tight convergence criteria such that the forces on the atoms were less than $2 \mathrm{meV} / \AA$ and the stresses were less than $0.1 \mathrm{meV} / \AA^{3}$. We have reproduced all the experimentally known phases of $\mathrm{P}$ and other low-lying phases, except for white-P and red-P, for which larger supercell calculations are necessary to describe the structure.

\section{B. Coupling and superconductivity calculations}

All superconductivity calculations are performed within SCDFT [66,67]. The approximations used have been described in previous works $[10,15,51,68,69]$. The pairing mechanism is due to the combined effect of electron-phonon coupling within DFT Kohn-Sham theory [70] as implemented in the QUANTUM ESPRESSO code and electronic screening computed in the static random-phase-approximation [71]. This allows us to calculate $T_{C}$ completely $a b$ initio, without introducing any empirical parameter such as the $\mu^{*}$ Coulomb pseudopotential usually used so solve the Eliashberg equations. Still, we can estimate an effective $\mu^{*}$ (reported in the Supplemental Material [50]), fitting the fully $a b$ initio $T_{C}$ with the Allen-Dynes-McMillan formula [72]. The electron-phonon coupling at the Fermi energy is described in the isotropic approximation by the Eliashberg spectral functions [52], defined as

$$
\alpha^{2} F(\omega)=\frac{1}{N_{E_{F}}} \sum_{\mathbf{k q}, v}\left|g_{\mathbf{k}, \mathbf{k}+\mathbf{q}, v}\right|^{2} \delta\left(\epsilon_{\mathbf{k}}\right) \delta\left(\epsilon_{\mathbf{k}+\mathbf{q}}\right) \delta\left(\omega-\omega_{\mathbf{q}, v}\right)
$$

where $N_{E_{F}}$ is the DOS at the Fermi level, $\omega_{\mathbf{q}, v}$ is the phonon frequency of mode $v$ at wave vector $\mathbf{q}$, and $\left|g_{\mathbf{k}, \mathbf{k}+\mathbf{q}, v}\right|$ is the electron-phonon matrix element between two electronic states with momenta $\mathbf{k}$ and $\mathbf{k}+\mathbf{q}$. All computed $\alpha^{2} F(\omega)$ are collected in Fig. 5. Anisotropy effects have been estimated to 
be irrelevant in the calculation of $T_{C}$ and are neglected in this work. Two significant moments of the Eliashberg function $\lambda$ and $\omega_{\log }$, defined as

$$
\begin{gathered}
\lambda=2 \int \frac{\alpha^{2} F(\omega)}{\omega} d \omega, \\
\omega_{\log }=\exp \left[\frac{2}{\lambda} \int \alpha^{2} F(\omega) \frac{\ln (\omega)}{\omega} d \omega\right],
\end{gathered}
$$

express, respectively, the electron-phonon coupling and the effective phononic energy.

Core atomic states are described in the norm-conserving pseudopotential approximation; valence state are described by a plane-wave basis set with an energy cutoff of $80 \mathrm{Ry}$. Since convergence checks have been performed independently on each phase, Brillouin zone integration is done with different sets of $k$ points in each crystalline structure, ranging from a minimum of $500 k$-points per unit reciprocal volume $\left(\mathrm{Bohr}^{3}\right)$ up to about $3000 k$-points per unit volume for electronic integration and about one-fourth of this density for phononic sampling. The strict convergence criteria ensure that the numerical error in the solution of the SCDFT equations is small as compared to the intrinsic error bar on the available functionals.

\section{ACKNOWLEDGMENTS}

The authors acknowledge the hospitality of the cini-Sardegna meeting, where part of this work was written. J.A.F.-L. acknowledges computational resources under Project s752 from the Swiss National Supercomputing Center (CSCS) in Lugano. A.D. and M.E. acknowledge the European Research Council with 2010-Advanced Grant No. 267777.
[1] A. P. Drozdov, M. I. Eremets, I. A. Troyan, V. Ksenofontov, and S. I. Shylin, Nature (London) 525, 73 (2015).

[2] I. I. Mazin, Nature (London) 525, 40 (2015).

[3] G. Eliashberg, Teor. Fiz. 38, 966 (1960); G. M. Eliashberg, Sov. Phys.-JETP 11, 696 (1960) [J. Exptl. Theoret. Phys. (U.S.S.R.) 38, 966 (1960)].

[4] I. Troyan, A. Gavriliuk, R. Rüffer, A. Chumakov, A. Mironovich, I. Lyubutin, D. Perekalin, A. P. Drozdov, and M. I. Eremets, Science 351, 1303 (2016).

[5] M. Einaga, M. Sakata, T. Ishikawa, K. Shimizu, M. I. Eremets, A. P. Drozdov, I. A. Troyan, N. Hirao, and Y. Ohishi, Nat. Phys. 12, 835 (2016).

[6] D. Duan, Y. Liu, F. Tian, D. Li, X. Huang, Z. Zhao, H. Yu, B. Liu, W. Tian, and T. Cui, Sci. Rep. 4, 6968 (2014).

[7] Y. Li, J. Hao, H. Liu, Y. Li, and Y. Ma, J. Chem. Phys. 140, 174712 (2014).

[8] N. Bernstein, C. S. Hellberg, M. D. Johannes, I. I. Mazin, and M. J. Mehl, Phys. Rev. B 91, 060511 (2015).

[9] I. Errea, M. Calandra, C. J. Pickard, J. Nelson, R. J. Needs, Y. Li, H. Liu, Y. Zhang, Y. Ma, and F. Mauri, Phys. Rev. Lett. 114, 157004 (2015).

[10] José A. Flores-Livas, A. Sanna, and E. K. U. Gross, Eur. Phys. J. B 89, 63 (2016).

[11] T. Ishikawa, A. Nakanishi, K. Shimizu, H. Katayama-Yoshida, T. Oda, and N. Suzuki, Sci. Rep. 6, 23160 (2016).

[12] C. Heil and L. Boeri, Phys. Rev. B 92, 060508 (2015).

[13] R. Akashi, W. Sano, R. Arita, and S. Tsuneyuki, Phys. Rev. Lett. 117, 075503 (2016)

[14] A. Drozdov, M. I. Eremets, and I. A. Troyan, arXiv:1508.06224.

[15] J. A. Flores-Livas, M. Amsler, C. Heil, A. Sanna, L. Boeri, G. Profeta, C. Wolverton, S. Goedecker, and E. K. U. Gross, Phys. Rev. B 93, 020508 (2016).

[16] A. Shamp, T. Terpstra, T. Bi, Z. Falls, P. Avery, and E. Zurek, arXiv: 1509.05455.

[17] M. Sakata, Y. Nakamoto, K. Shimizu, T. Matsuoka, and Y. Ohishi, Phys. Rev. B 83, 220512 (2011).

[18] E. Wigner and H. B. Huntington, J. Chem. Phys. 3, 764 (1935).

[19] N. Ashcroft, Phys. Rev. Lett. 21, 1748 (1968).

[20] M. I. Eremets, I. A. Troyan, and A. P. Drozdov, arXiv:1601.04479.

[21] R. P. Dias and I. F. Silvera, Science 355, 715 (2017).
[22] A. F. Goncharov and V. V. Struzhkin, arXiv:1702.04246.

[23] P. Loubeyre, F. Occelli, and P. Dumas, arXiv:1702.07192.

[24] M. I. Eremets and A. P. Drozdov, arXiv:1702.05125.

[25] E. G. Brovman, Yu. Kagan, and A. Kholas, Sov. Phys. JETP 34 1300 (1972).

[26] M. Eremets and I. Troyan, Nat. Mater. 10, 927 (2011).

[27] P. Dalladay-Simpson, R. T. Howie, and E. Gregoryanz, Nature (London) 529, 63 (2016).

[28] C. Buzea and K. Robbie, Supercond. Sci. Technol. 18, R1 (2004).

[29] R. Zhang, J. Waters, A. K. Geim, and I. V. Grigorieva, Nat. Commun. 8, 15036 (2017).

[30] H. Kawamura, I. Shirotani, and K. Tachikawa, Solid State Commun. 54, 775 (1985).

[31] M. Karuzawa, M. Ishizuka, and S. Endo, J. Phys.: Condens. Matter 14, 10759 (2002).

[32] J. Guo, H. Wang, F. von Rohr, W. Yi, Y. Zhou, Z. Wang, S. Cai, S. Zhang, X. Li, Y. Li, J. Liu, K. Yang, A. Li, S. Jiang, Q. Wu, T. Xiang, R. J. Cava, and L. Sun, arXiv:1611.03330.

[33] M. Ruck, D. Hoppe, B. Wahl, P. Simon, Y. Wang, and G. Seifert, Angew. Chem. 117, 7788 (2005).

[34] R. Hultgren, N. Gingrich, and B. Warren, J. Chem. Phys. 3, 351 (1935).

[35] K. Shimizu, K. Amaya, and N. Suzuki, J. Phys. Soc. Jpn. 74, 1345 (2005).

[36] Y. Akahama, H. Kawamura, S. Carlson, T. Le Bihan, and D. Häusermann, Phys. Rev. B 61, 3139 (2000).

[37] J. C. Jamieson, Science 139, 1291 (1963).

[38] M. Marqués, G. J. Ackland, L. F. Lundegaard, S. Falconi, C. Hejny, M. I. McMahon, J. Contreras-García, and M. Hanfland, Phys. Rev. B 78, 054120 (2008).

[39] A. Nakanishi, T. Ishikawa, H. Nagara, K. Shimizu, and H. Katayama-Yoshida, High Pressure Res. 32, 3 (2012).

[40] T. Sugimoto, Y. Akahama, H. Fujihisa, Y. Ozawa, H. Fukui, N. Hirao, and Y. Ohishi, Phys. Rev. B 86, 024109 (2012).

[41] Y. Akahama, M. Kobayashi, and H. Kawamura, Phys. Rev. B 59, 8520 (1999).

[42] F. J. H. Ehlers and N. E. Christensen, Phys. Rev. B 69, 214112 (2004).

[43] T. Ishikawa, H. Nagara, K. Kusakabe, and N. Suzuki, Phys. Rev. Lett. 96, 095502 (2006). 
[44] H. Fujihisa, Y. Akahama, H. Kawamura, Y. Ohishi, Y. Gotoh, H. Yamawaki, M. Sakashita, S. Takeya, and K. Honda, Phys. Rev. Lett. 98, 175501 (2007).

[45] H. Katzke and P. Tolédano, Phys. Rev. B 77, 024109 (2008).

[46] S. E. Boulfelfel, G. Seifert, Y. Grin, and S. Leoni, Phys. Rev. B 85, 014110 (2012).

[47] A. Sanna, A. V. Fedorov, N. I. Verbitskiy, J. Fink, C. Krellner, L. Petaccia, A. Chikina, D. Y. Usachov, A. Grüneis, and G. Profeta, 2D Mater. 3, 025031 (2016).

[48] K. T. Chan, B. D. Malone, and M. L. Cohen, Phys. Rev. B 88, 064517 (2013).

[49] I. Lifshitz, J. Exp. Theor. Phys. 11, 1130 (1960) [ZhETF, 38 (5), 1569 (Nov. 1960)].

[50] See Supplemental Material at http://link.aps.org/supplemental/ 10.1103/PhysRevMaterials.1.024802 for detailed spectral function and analysis of the Lifshitz transition.

[51] M. Monni, F. Bernardini, A. Sanna, G. Profeta, and S. Massidda, Phys. Rev. B 95, 064516 (2017).

[52] P. B. Allen and B. Mitrović, Theory of Superconducting Tc, Solid State Physics Vol. 37 (Academic Press, New York, 1983), pp. 1-92.

[53] Z. P. Yin, F. Gygi, and W. E. Pickett, Phys. Rev. B 80, 184515 (2009).

[54] J. A. Flores-Livas, R. Debord, S. Botti, A. San Miguel, M. A. L. Marques, and S. Pailhès, Phys. Rev. Lett. 106, 087002 (2011).

[55] S. Goedecker, J. Chem. Phys. 120, 9911 (2004).

[56] M. Amsler and S. Goedecker, J. Chem. Phys. 133, 224104 (2010).

[57] M. Amsler, J. A. Flores-Livas, L. Lehtovaara, F. Balima, S. A. Ghasemi, D. Machon, S. Pailhès, A. Willand, D. Caliste, S. Botti, A. San Miguel, S. Goedecker, and M. A. L. Marques, Phys. Rev. Lett. 108, 065501 (2012).
[58] J. A. Flores-Livas, M. Amsler, T. J. Lenosky, L. Lehtovaara, S. Botti, M. A. L. Marques, and S. Goedecker, Phys. Rev. Lett. 108, 117004 (2012).

[59] M. Amsler, J. A. Flores-Livas, T. D. Huan, S. Botti, M. A. L. Marques, and S. Goedecker, Phys. Rev. Lett. 108, 205505 (2012).

[60] S. Botti, J. A. Flores-Livas, M. Amsler, S. Goedecker, and M. A. L. Marques, Phys. Rev. B 86, 121204 (2012).

[61] S. Roy, S. Goedecker, M. J. Field, and E. Penev, J. Phys. Chem. B 113, 7315 (2009).

[62] M. Sicher, S. Mohr, and S. Goedecker, J. Chem. Phys. 134, 044106 (2011).

[63] F. Jensen, Introduction to Computational Chemistry, 2nd ed. (John Wiley, New York, 2011).

[64] J. P. Perdew, K. Burke, and M. Ernzerhof, Phys. Rev. Lett. 77, 3865 (1996).

[65] G. Kresse and J. Furthmüller, Comput. Mater. Sci. 6, 15 (1996).

[66] L. N. Oliveira, E. K. U. Gross, and W. Kohn, Phys. Rev. Lett. 60, 2430 (1988).

[67] M. Lüders, M. A. L. Marques, N. N. Lathiotakis, A. Floris, G. Profeta, L. Fast, A. Continenza, S. Massidda, and E. K. U. Gross, Phys. Rev. B 72, 024545 (2005).

[68] J. A. Flores-Livas and A. Sanna, Phys. Rev. B 91, 054508 (2015).

[69] A. Linscheid, A. Sanna, A. Floris, and E. K. U. Gross, Phys. Rev. Lett. 115, 097002 (2015).

[70] S. Baroni, S. de Gironcoli, A. Dal Corso, and P. Giannozzi, Rev. Mod. Phys. 73, 515 (2001).

[71] S. Massidda, F. Bernardini, C. Bersier, A. Continenza, P. Cudazzo, A. Floris, H. Glawe, M. Monni, S. Pittalis, G. Profeta, A. Sanna, S. Sharma, and E. K. U. Gross, Supercond. Sci. Technol. 22, 034006 (2009).

[72] P. B. Allen and R. C. Dynes, Phys. Rev. B 12, 905 (1975). 